\title{
Autism Spectrum Disorder Diagnosis in Diagnostic and Statistical Manual of Mental Disorders-5 Compared to Diagnostic and Statistical Manual of Mental Disorders-IV
}

\author{
Yun Shin Lim, Kee Jeong Park, and Hyo-Won Kim \\ Department of Psychiatry, University of Ulsan College of Medicine, Asan Medical Center, Seoul, Korea
}

\begin{abstract}
Objectives: The objective of this study was to investigate the concordance of Diagnostic and Statistical Manual of Mental Disorders (DSM-IV and DSM-5) diagnostic criteria for autism spectrum disorder (ASD).

Methods: We retrospectively reviewed the medical records of 170 subjects (age range: 3-23, 140 boys) with developmental delay or social deficit from January 2011 to July 2016 at the Department of Psychiatry of Asan Medical Center. The Autism Diagnostic Interview-Revised (ADI-R), the Autism Diagnostic Observation Schedule (ADOS), and intelligence tests were performed for each subject. Diagnosis was reviewed and confirmed for each subject with DSM-IV Pervasive Developmental Disorder (PDD) and DSM-5 ASD criteria, respectively.

Results: Fifty-eight of 145 subjects (34.1\%) who were previously diagnosed as having PDD in DSM-IV did not meet DSM-5 ASD criteria. Among them, 28 (48.3\%) had Asperger's disorder based on DSM-IV. Most algorithm scores on ADOS and all algorithm scores on ADI-R were highest in subjects who met both DSM-IV PDD criteria and DSM-5 ASD criteria (the Convergent group), followed by subjects with a DSM-IV PDD diagnosis who did not have a DSM-5 ASD diagnosis (the Divergent group), and subjects who did not meet either DSM-IV PDD or DSM-5 ASD criteria (the non-PDD group). Intelligence quotient was lower in the Convergent group than in the Divergent group.

Conclusion: The results of our study suggest that ASD prevalence estimates could be lower under DSM-5 than DSM-IV diagnostic criteria. Further prospective study on the impact of new DSM-5 ASD diagnoses in Koreans with ASD is needed.
\end{abstract}

Key Words: Autism spectrum disorder; Diagnostic and Statistical Manual of Mental Disorders-IV;

Diagnostic and Statistical Manual of Mental Disorders-5; pervasive developmental disorder.

Received: June 20, 2018 / Revision: August 13, 2018 / Accepted: August 24, 2018

Address for correspondence: Hyo-Won Kim, Department of Psychiatry, University of Ulsan College of Medicine, Asan Medical Center, 88 Olympic-ro 43-gil, Songpa-gu, Seoul 05505, Korea

Tel: +82-2-3010-3414, Fax: +82-2-485-8381, E-mail: shingubi@amc.seoul.kr

\section{INTRODUCTION}

Autism spectrum disorder (ASD) is a neurodevelopmental disorder characterized by qualitative deficits in social communication and social interactions, and restricted patterns of behaviors, interests, and activities [1]. The prevalence of ASD has been rising; the latest Centers for Disease Control survey found a prevalence of one per 59 persons in the USA [2], and the global prevalence is estimated to be $0.6-1.8 \%$. A study of Korean children in one community reported a high prevalence of $2.64 \%$, and has drawn social attention [3].

The revised Diagnostic and Statistical Manual of Mental Disorders, Fifth Edition (DSM-5) [4], published in 2013, in-

This is an Open Access article distributed under the terms of the Creative Commons Attribution Non-Commercial License (https://creativecommons.org/licenses/by-nc/4.0) which permits unrestricted non-commercial use, distribution, and reproduction in any medium, provided the original work is properly cited. cluded major changes in the diagnostic criteria for ASD. First, autistic disorder, childhood disintegrative disorder, Asperger's disorder, and pervasive developmental disorder not otherwise specified (PDD-NOS), which were previously subcategories of PDD, were combined under the single diagnosis of ASD. These disorders are now considered as a continuous spectrum of ASD. Second, the core symptoms of ASD were reduced to two domains, with deficits in social communication and social interaction merged into a single domain, and restricted interests and repetitive patterns of behaviors as the second domain. Third, the age of onset from 'prior to age 3 years' in DSM-IV was changed to symptoms present 'in the early developmental period.' Fourth, the new diagnosis of social communication disorder (SCD) was added, for individuals who mostly show persistent deficits in pragmatic communication, without restricted or repetitive behaviors [4]. 
Since the draft of the DSM-5 was published, there has been heated debate about these diagnostic criteria. In particular, only $52-76 \%$ of children diagnosed with PDD based on the DSM-IV [5] would be diagnosed with ASD in DSM-5 [6], and patients with high cognitive function, or those previously diagnosed with Asperger's disorder, would be less likely to be diagnosed with ASD under the DSM- 5 criteria $[6,7]$.

Kim et al. [3,7] found that most children diagnosed with autistic disorder (99\%), Asperger's disorder (92\%), or PDDNOS (63\%) based on DSM-IV met the diagnostic criteria of DSM- 5 , but that $8 \%$ of children diagnosed with Asperger's disorder and 32\% of children diagnosed with PDD-NOS did not meet the criteria for ASD and would instead be diagnosed with SCD. However, as a community-based survey, this study was not able to show how children diagnosed with PDD under DSM-IV are being diagnosed under DSM-5 in an actual clinical setting. Moreover, most other studies have investigated Western children with ASD. In particular, there are no studies examining the concordance between DSMIV and DSM-5 diagnoses in a Korean clinical population. Therefore, in this study, we aimed to investigate the concordance between DSM-IV and DSM-5 diagnoses in a clinical population in Korea, and to examine clinical characteristics related to the changes in the diagnoses.

\section{METHODS}

\section{Subjects and methods}

The subjects in this study consisted of 170 patients who had visited the Department of Pediatric Psychiatry at Asan Medical Center between January 2011 and July 2016 due to a history of developmental delay. The inclusion criteria were as follow: 1) a child, youth, or adult aged 3-23 years old, 2) on whom were performed an Autism Diagnostic Interview-Revised (ADI-R), an Autism Diagnostic Observation Schedule (ADOS), and an intelligence test. Patients with a congenital genetic disorder, cerebral palsy, neurological disorder, or epilepsy were excluded. The medical records were reviewed retrospectively by a pediatric psychiatrist, and ASD was diagnosed based on DSM-IV and DSM-5 diagnostic criteria.

The study was approved by the Asan Medical Center Institutional Review Board (IRB No. 2018-0630).

\section{Assessments}

\section{Autism Diagnostic Interview-Revised (ADI-R)}

This is an interview-based test designed for the diagnosis of ASD based on DSM-IV, DSM-5, and International Classification of Diseases, 10th revision (ICD-10) diagnostic criteria; it is able to differentiate ASD from other developmental dis- orders. The interview is conducted with the patient's primary caregiver and covers the four domains of social interaction; language and communication; restricted, repetitive, and stereotyped patterns of behavior; and age of onset. This test is able to collect information describing characteristic behaviors in detail, which is necessary for a differential diagnosis of ASD from other types of developmental disorder. It is used as a complementary instrument with ADOS, in which the patient's behaviors are assessed by direct observation [8].

\section{Autism Diagnostic Observation Schedule (ADOS)}

This is a semi-structured, standardized test for the diagnosis of ASD based on DSM-IV, DSM-5, and ICD-10 diagnostic criteria. A diagnosis is reached by scoring ASD-related behaviors with direct observation and assessment through semistructured play and activities. The patient is observed and assessed comprehensively across the five subdomains of language and communication, reciprocal social interaction, imagination and creativity, as well as stereotyped behaviors and restricted interests and other abnormal behaviors. Age and verbal ability are not limitations of the tests. Instead, different modules can be selected to suit the patient's age, verbal ability, and intelligence, from non-verbal patients (module 1) to adults with fluent verbal ability (module 4) [9].

\section{Intelligence tests}

Korean-Wechsler Preschool and Primary Scale of Intelligence (K-WPPSI)

This test was standardized to suit the circumstances by the Korean Institute for Developmental Tests, and is designed to measure intelligence in individual children between 3 years of age and 7 years, 3 months. As an intelligence test developed for preschool and young primary school children who are too young for the Wechsler Intelligence Scale for Children (WISC), the Korean-Wechsler Preschool and Primary Scale of Intelligence (K-WPPSI) consists of two subscales for Performance Intelligence Quotient (PIQ) and Verbal Intelligence Quotient (VIQ). Each subscale consists of six subtests, and the total result is given as the full scale intelligence quotient (FSIQ) [10].

Korean-Wechsler Intelligence Scale for Children-III (K-WISC-III)

This is a Korean standardized intelligence test adapted from WISC to suit the circumstances in South Korea. The test allows for clinical assessment of intelligence in individual children aged from 6 years to 16 years, 11 months.

The K-WISC-III measures intelligence through various subtests that evaluate different abilities. In addition to PIQ, 
VIQ, and FSIQ, the test provides index scores for four factors based on factor analysis. Compared to the previous edition, the K-WISC-III consists of 13 subtests, adding symbol search to the previous 12 subtests [11].

Korean Wechsler Intelligence Scale for Children-4th edition (K-WISC-IV)

This test is a revision of the K-WISC-III, which is used in children and youths aged from 6 years to 16 years, 11 months. The K-WISC-IV not only gives FISQ as a measure of overall intelligence, but also includes subtests and index scores for specific cognitive abilities. Compared to the previous version, the K-WISC-IV adds five new subtests (picture concepts, letter-number sequencing, matrix reasoning, word reasoning, and cancellation), making a total of 15 subtests. The K-WISCIV provides four index scores, and including FSIQ like the previous version. The terms VIQ and PIQ were replaced with verbal comprehension index and perceptual reasoning index, respectively [12].

Korean Wechsler Adult intelligence scale-IV (K-WAIS-IV)

This is a standardized Korean adaptation of the Wechsler Adult intelligence scale (WAIS)-IV, which was revised in 2008 in the USA. The K-WAIS-IV was developed in 2012 to suit the circumstances in South Korea. Theseday, it is frequently used as a test of intelligence in adults. The test provides a clinical assessment of cognitive ability in youths or adults aged from 16 to 69 years. In contrast to the previous scoring structure, dividing scores into VIQ and PIQ, the KWAIS-IV was changed to include four indexes (verbal comprehension, perceptual reasoning, working memory, and processing speed), in addition to FSIQ, as in the previous version [13].

\section{Statistical analysis}

All 170 subjects were divided into a PDD group and a nonPDD group based on a review of their medical records using the DSM-IV diagnostic criteria. Categorical variables were compared between the two groups using the $\chi^{2}$ test, and continuous variables were compared using the independent t-test.

In addition, we compared variables between the group that met both DSM-IV PDD criteria and DSM-5 ASD criteria (the Convergent group), the group that had been diagnosed with PDD under DSM-IV criteria but were not included in the ASD diagnostic criteria in DSM-5 (the Divergent group), and the non-PDD group. Categorical variables were compared using the $\chi^{2}$ test, and continuous variables were compared using analysis of variance. For post-hoc analysis, Duncan's posthoc test was used.
In this study, direct comparison of intelligence scores was problematic because different intelligence tests were used depending on chronological age. Therefore, we converted FSIQ into a categorical variable with three possible values $(\geq 80$ : normal, 70-79: borderline, $\geq 69$ : mental retardation).

We conducted the analysis using the statistical program SPSS 20 for Windows (IBM Corp., Armonk, NY, USA). Statistical significance for all other comparisons was defined as $\mathrm{p}<0.05$; all comparisons were two-tailed.

\section{RESULTS}

\section{Demographic characteristics (Table 1)}

In this study, 145 patients were diagnosed with PDD under the DSM-IV diagnostic criteria (PDD group, mean age: $9.8 \pm 5.8$ years), while 25 patients were not diagnosed with PDD (non-PDD group, mean age: $13.7 \pm 5.2$ years). The nonPDD group was significantly older than the PDD group $(\mathrm{p}=$ 0.002). There was no significant difference between the two groups in gender distribution. Compared to the non-PDD group, the PDD group showed a significantly higher number of patients with mental retardation $(\mathrm{p}=0.003)$.

In the PDD group, there were 92 patients (63.4\%) with autism disorder, 38 patients (26.3\%) with Asperger's disorder, and 15 patients (10.3\%) with PDD-NOS. In the PDD group,

Table 1. Demographic characteristics for participants with and without DSM-IV PDD

\begin{tabular}{lcccc}
\hline & $\begin{array}{c}\text { PDD } \\
(\mathrm{n}=145)\end{array}$ & $\begin{array}{c}\text { Non-PDD } \\
(\mathrm{n}=25)\end{array}$ & $\chi^{2}$ & $\mathrm{p}$ \\
\hline $\begin{array}{l}\text { Age, year, mean } \pm \text { SD } \\
\text { Gender (n, \%) }\end{array}$ & $9.8 \pm 5.8$ & & 3.152 & 0.002 \\
$\quad$ Male & & & 0.002 & 0.968 \\
FSIQ (n, \%) & $128(88.3)$ & $22(88.0)$ & & \\
$\quad$ Normal & & & 11.407 & 0.003 \\
Borderline & $66(53.7)$ & $13(56.5)$ & & \\
Mental Retardation & $43(11.4)$ & $8(34.8)$ & & \\
DSM-IV Diagnosis (n, \%) & & $2(8.7)$ & & \\
$\quad$ Autistic disorder & $92(63.4)$ & & & \\
Asperger's disorder & $38(26.3)$ & & & \\
PDD-NOS & $15(10.3)$ & & & \\
ADOS Module (n, \%) & & & 12.261 & 0.007 \\
Module 1 & $5(3.4)$ & $1(4.0)$ & & \\
Module 2 & $57(39.3)$ & $1(4.0)$ & & \\
Module 3 & $48(33.1)$ & $12(48.0)$ & & \\
Module 4 & $35(24.1)$ & $11(44.0)$ & & \\
\hline
\end{tabular}

Intelligence Quotient ratings are as follow, normal is 80 and above; borderline is 70-79; mental retardation is 69 and below. ADOS: Autism Diagnostic Observation Schedule, DSM: Diagnostic and Statistical Manual of Mental Disorders, FSIQ: full scale intelligence quotient, NOS: not otherwise specified, PDD: pervasive developmental disorder, SD: standard deviation 
5 patients (3.4\%) were tested using ADOS module 1, 57 patients (39.3\%) were tested using module 2, 48 patients (33.1\%) were tested using module 3 , and 35 patients $(24.1 \%)$ were tested using module 4 . In the non-PDD group, 1 patient (4.0\%) was tested using module 1,1 patient (4.0\%) was tested using module 2, 12 patients (48.0\%) were tested using module 3, and 11 patients (44.0\%) were tested using module 4 . The difference in the distribution of ADOS modules between the two groups was statistically significant $(p=0.007)$.

\section{Concordance of diagnoses based on DSM-5 criteria in the PDD group and the non-PDD group (Table 2)}

There were 87 patients (51.2\%) who were diagnosed with PDD in DSM-IV and were also diagnosed with ASD when the DSM-5 ASD diagnostic criteria were applied; there were 58 patients (34.1\%) who were diagnosed with PDD in DSMIV, but were not diagnosed with ASD based on the DSM-5 criteria. Meanwhile, there were 25 patients (14.7\%) who did not meet either the DSM-IV PDD criteria or the DSM-5 ASD criteria, and there were no patients who were not diagnosed with PDD in DSM-IV but were newly diagnosed with ASD in DSM-5.

\section{Comparison of sub-diagnoses in DSM-IV in the Con-}

Table 2. DSM-5 diagnoses of participants with and without DSMIV PDD

\begin{tabular}{lcc}
\hline & PDD $(n, \%)$ & Non-PDD $(n, \%)$ \\
\hline ASD & $87(51.2)$ & $0(0.0)$ \\
Non-ASD & $58(34.1)$ & $25(14.7)$ \\
\hline
\end{tabular}

ASD: autism spectrum disorder, DSM: Diagnostic and Statistical Manual of Mental Disorders, PDD: pervasive developmental disorder vergent group and the Divergent group (Table 3)

Among patients diagnosed with autistic disorder, Asperger's disorder, and PDD-NOS in DSM-IV, 79.3\%, 26.3\%, and $26.7 \%$, respectively, satisfied the diagnostic criteria for ASD in DSM-5. (the Convergent group). Meanwhile, 20.7\%, 73.7\%, and $73.3 \%$ of these patients did not meet the ASD criteria in DSM-5 (the Divergent group). The Asperger's disorder and PDD-NOS patients were significantly less likely to meet the ASD diagnostic criteria in DSM-5 ( $\mathrm{p}<0.001)$.

In addition, when the DSM-5 diagnostic criteria were applied to the 58 patients in the Divergent group, 55 patients (94.8\%) satisfied the criteria for SCD, which was newly added in DSM-5, while two patients were diagnosed with mild intellectual disability, and 1 patient was diagnosed with borderline intellectual functioning and attention-deficit/hyperactivity disorder.

\section{Comparison of intelligence quotients between the Convergent group and the Divergent group (Table 4)}

Among patients with normal intelligence, borderline intellectual functioning, and mental retardation, the proportions of these patients in the Convergent group were $40.8 \%$, $10.5 \%$, and $48.7 \%$, respectively. The proportions in the Divergent group were $73.5 \%, 14.3 \%$, and $12.2 \%$, respectively. The Divergent group included more patients with normal intelligence $(\mathrm{p}<0.001)$.

\section{Comparison of ADI-R and ADOS results between different diagnoses in DSM-IV and DSM-5 (Table 5)}

In Table 5, ADI-R and ADOS results are compared among the Convergent group, the Divergent group, and the non-

Table 3. DSM-IV diagnosis of Convergent and Divergent groups

\begin{tabular}{lcc}
\hline & Convergent group $(\mathrm{n}, \%)$ & Divergent group $(\mathrm{n}, \%)$ \\
\hline DSM-IV diagnosis & & $\chi^{2}$ \\
Autistic disorder & $73(79.3)$ & $19(20.7)$ \\
Asperger's disorder & $10(26.3)$ & $28(73.7)$ \\
PDD-NOS & $4(26.7)$ & $11(73.3)$
\end{tabular}

The Convergent group has both DSM-IV PDD diagnosis and DSM-5 ASD diagnosis. The Divergent group has a DSM-IV PDD diagnosis and does not have a DSM-5 ASD diagnosis. ASD: autism spectrum disorder, DSM: Diagnostic and Statistical Manual of Mental Disorders, NOS: not otherwise specified, PDD: pervasive developmental disorder

Table 4. Comparisons of FSIQ between Convergent and Divergent groups

\begin{tabular}{lcc}
\hline & Convergent group $(\mathrm{n}, \%)$ & Divergent group $(\mathrm{n}, \%)$ \\
\hline FSIQ & $31(40.8)$ & \\
Normal & $8(10.5)$ & $36(73.5)$ \\
Borderline & $37(48.7)$ & $7(14.3)$ \\
Mental retardation & $6(12.2)$ \\
\hline
\end{tabular}

The Convergent group has both DSM-IV PDD diagnosis and DSM-5 ASD diagnosis. The Divergent group has a DSM-IV PDD diagnosis and does not have a DSM-5 ASD diagnosis. Intelligence Quotient ratings are as follow, normal is 80 and above; borderline is 70-79; mental retardation is 69 and below. ASD: autism spectrum disorder, DSM: Diagnostic and Statistical Manual of Mental Disorders, FSIQ: full scale intelligence quotient, PDD: pervasive developmental disorder 
Table 5. Comparisons of the ADOS and ADI-R algorithm scores between Convergent group and Divergent group

\begin{tabular}{|c|c|c|c|c|c|c|}
\hline & $\begin{array}{c}\text { Convergent } \\
\text { group }^{a}(\mathrm{n}=87)\end{array}$ & $\begin{array}{c}\text { Divergent } \\
\text { group }^{\mathrm{b}}(\mathrm{n}=58)\end{array}$ & $\begin{array}{c}\text { Non-PDD } \\
(n=25)\end{array}$ & $\mathrm{F}$ & $\mathrm{p}$ & $\begin{array}{l}\text { Post hoc test } \\
\text { (Duncan) }\end{array}$ \\
\hline \multicolumn{7}{|l|}{ ADI-R algorithm scores } \\
\hline $\begin{array}{l}\text { Qualitative abnormalities in reciprocal social } \\
\text { interaction }\end{array}$ & $23.06 \pm 4.04$ & $14.52 \pm 4.90$ & $4.88 \pm 4.56$ & 179.72 & $<0.001$ & $c<b<a$ \\
\hline \multicolumn{7}{|l|}{ Qualitative abnormalities in communication } \\
\hline Verbal communication & $17.43 \pm 3.44$ & $11.43 \pm 3.49$ & $2.60 \pm 2.81$ & 199.10 & $<0.001$ & $c<b<a$ \\
\hline Non-verbal communication & $11.38 \pm 2.87$ & $6.64 \pm 2.86$ & $1.52 \pm 1.94$ & 140.76 & $<0.001$ & $c<b<a$ \\
\hline $\begin{array}{l}\text { Restricted, repetitive and stereotyped } \\
\text { patterns of behavior }\end{array}$ & $5.14 \pm 2.14$ & $2.90 \pm 2.08$ & $0.64 \pm 0.76$ & 57.67 & $<0.001$ & $c<b<a$ \\
\hline Onset $<36$ months & $4.61 \pm 0.81$ & $3.34 \pm 1.84$ & $0.36 \pm 1.08$ & 106.62 & $<0.001$ & $c<b<a$ \\
\hline \multicolumn{7}{|l|}{ ADOS algorithm scores } \\
\hline Communication & $6.78 \pm 2.28$ & $4.50 \pm 1.76$ & $1.40 \pm 1.71$ & 73.10 & $<0.001$ & $c<b<a$ \\
\hline Reciprocal social interaction & $11.17 \pm 2.78$ & $8.47 \pm 2.80$ & $3.12 \pm 2.91$ & 81.93 & $<0.001$ & $c<b<a$ \\
\hline Imagination \& creativity & $2.21 \pm 1.99$ & $2.28 \pm 2.86$ & $1.12 \pm 0.83$ & 2.70 & 0.038 & $c<a=b$ \\
\hline Stereotyped behaviors and restricted interests & $2.28 \pm 1.62$ & $1.43 \pm 1.64$ & $0.28 \pm 0.68$ & 17.83 & $<0.001$ & $c<b<a$ \\
\hline
\end{tabular}

Data are mean \pm SD values. The Convergent group has both DSM-IV PDD diagnosis and DSM-5 ASD diagnosis. The Divergent group has a DSM-IV PDD diagnosis and does not have a DSM-5 ASD diagnosis. ADOS: Autism Diagnostic Observation Schedule, ADI-R: Autism Diagnostic Interview-Revised, ASD: autism spectrum disorder, DSM: Diagnostic and Statistical Manual of Mental Disorders, PDD: pervasive developmental disorder, SD: standard deviation

PDD group. The three groups showed statistically significant differences in the ADI-R in the domains of qualitative abnormalities in reciprocal social interaction, qualitative abnormalities in verbal communication, qualitative abnormalities in non-verbal communication, restricted, repetitive and stereotyped patterns of behavior, and onset $<36$ months (all $\mathrm{p}<0.001)$. These differences were confirmed in post-hoc analysis, with the Convergent group showing the highest scores in all domains, followed by the Divergent group, then the nonPDD group.

Statistically significant differences among the three groups were also observed in the ADOS domains of communication, reciprocal social interaction, stereotyped behavior, and restricted interests (all $\mathrm{p}<0.001$ ). The Convergent group showed the highest scores in all domains, followed by the Divergent group, then the non-PDD group. However, in the imagination/creativity domain, the Divergent group showed the highest score (2.21 \pm 1.99$)$, followed by the Convergent group (2.28 \pm $2.86)$, then the non-PDD group (1.12 \pm 0.83$)$, but these differences were not statistically significant.

\section{DISCUSSION}

We examined the concordance between diagnoses with DSM-IV and the revised DSM-5 based on a retrospective chart review, and we compared clinical characteristics of subjects. In this study, 58 patients (34.1\%) were diagnosed with PDD based on the DSM-IV criteria, but did not meet the diagnostic criteria for ASD in DSM-5. Among DSM-IV sub- diagnoses, the subjects who were not diagnosed with DSM5 ASD criteria were most common in Asperger's disorder (28 patients; 48.3\%). When compared clinical characteristics of Convergent, Divergent, and non-PDD groups based on their diagnoses in DSM-IV and DSM-5, we observed statistically significant differences in most domains in the ADI-R and ADOS (excluding only imagination/creativity), as well as in FSIQ.

We observed about one-thirds ofpatient who was diagnosed with PDD based on DSM-IV criteria, but was not diagnosed with ASD based on the revised DSM- 5 criteria. In DSM-5, the sub-diagnoses of PDD are combined under the single diagnosis of ASD, and the diagnostic criteria for ASD are newly defined. However, due to these alterations in the DSM-5 criteria, when patients are re-evaluated and given a different diagnosis, or excluded from the ASD diagnosis, this change not only causes confusion for the patient and their family, but is also likely to have a major impact on research on ASD [14]. Moreover, early screening and intervention are very important in ASD, but patients who are excluded from the DSM-5 diagnosis are expected to lose proper therapeutic support or have difficulty receiving support from medical systems or the government. In this regard, further research and discussion will be required regarding special education, therapeutic interventions, and social support for these patients.

In this study, among PDD sub-diagnoses, the percentage of patients who did not meet the ASD diagnostic criteria in DSM-5 was highest for Asperger's disorder. This is consistent with previous studies that applied the DSM- 5 criteria to 
children diagnosed with PDD in DSM-IV and reported low sensitivity for individuals with Asperger's disorder or highfunctioning PDD, with only $42 \%$ re-diagnosed with ASD in DSM-5 $[15,16]$.

We divided subjects into Convergent, Divergent, and nonPDD groups, and compared clinical characteristics in relation to the diagnostic differences in DSM-IV and DSM-5. We observed significant differences among the three groups in the all domains of the ADI-R and in three domains of ADOS (excluding only imagination/creativity). Previous study showed ADI-R and ADOS scores were higher in a group diagnosed with PDD and ASD in both DSM-IV and DSM-5 than in a group that was diagnosed with PDD in DSM-IV but not ASD in DSM-5, is consistent with our findings [7]. In addition, the Convergent and Divergent groups showed no significant difference in the imagination/creativity domain of ADOS in this study. This indicates that, even though the Divergent group was often excluded from the revised ASD diagnostic criteria in DSM-5, they still show significant deficits or low levels of imaginative, creative, and pretend play. More detailed studies will be needed to further investigate this finding.

We also observed clear differences between the Convergent group and the Divergent group in the distribution of intelligence. Specifically, the Divergent group showed a higher rate of patients with normal intelligence. This results are also in line with previous studies, which reported that individuals with mental retardation are more likely to meet the ASD diagnostic criteria in DSM-5 compared to those without mental retardation $[15,17]$.

Of the 58 patients diagnosed with PDD in DSM-IV but not ASD in DSM- 5 in our study, 55 patients (94.8\%) fit the definition of SCD. SCD is characterized by problems in the ability to use language properly suited to the social situation and the context, in reciprocal conversation, and in understanding non-verbal interactions and hidden meanings in language. However, there is room for debate as to whether SCD is simply a form of mild ASD without restricted interests, repetitive behaviors, and sensory abnormalities, or whether it should be considered an independent disease [18]. Further studies are needed in this area, as there have not yet been enough prospective follow-up studies or genetic studies to show whether SCD is an independent disease.

This study has several limitations. First, there were significant differences between the PDD and non-PDD group in age and distribution of ADOS modules. In future studies, it will be necessary to account for such differences from the subject recruitment stage. Second, since there are only 170 subjects in the study, it is difficult to generalize the results. Third, because the study was based on retrospective chart reviews, there were limits to the clinical information that could be obtained. Indeed, of the subjects in the study, there were 11 patients in the Convergent group and 9 patients in the Divergent group without results for intelligence tests. Fourth, because this was a cross-sectional study, causality cannot be clearly elucidated. In the future, it will be necessary to verify the results of our study in a more systematically designed prospective follow-up study.

\section{CONCLUSION}

The results of our study suggest that children and adolescents diagnosed with PDD using the DSM-IV diagnostic criteria were less likely to be diagnosed with ASD using the DSM5 diagnostic criteria. This study has importance in that we examined changes in diagnoses using DSM-IV compared to the revised diagnostic criteria of DSM-5, which was published 20 years after the DSM-IV, and investigated the clinical characteristics of these patients.

\section{Acknowledgments}

This research was supported by Basic Science Research Program through the National Research Foundation of Korea (NRF) funded by the Ministry of Science and ICT (No. 2018R1A2B6002216).

\section{Conflicts of Interest}

The authors have no financial conflicts of interest.

\section{REFERENCES}

1) Wong CM, Koh HC. Brief report: investigating the implications of applying the new DSM-5 criteria for diagnosing autism spectrum disorder in a preschool population in Singapore. J Autism Dev Disord 2016;46:3177-3182.

2) Baio J, Wiggins L, Christensen DL, Maenner MJ, Daniels J, Warren Z, et al. Prevalence of autism spectrum disorder among children aged 8 years - autism and developmental disabilities monitoring network, 11 sites, United States, 2014. MMWR Surveill Summ 2018; 67:1-23.

3) Kim YS, Leventhal BL, Koh YJ, Fombonne E, Laska E, Lim EC, et al. Prevalence of autism spectrum disorders in a total population sample. Am J Psychiatry 2011;168:904-912.

4) American Psychiatric Association. Diagnostic and statistical manual of mental disorders-5. Arlington: American Psychiatric Publishing;2013.

5) American Psychiatric Association. Diagnostic and statistical manual of mental disorders-IV. 4th ed. Washington D.C: American Psychiatric Association;1994.

6) Mattila ML, Kielinen M, Linna SL, Jussila K, Ebeling H, Bloigu R, et al. Autism spectrum disorders according to DSM-IV-TR and comparison with DSM-5 draft criteria: an epidemiological study. J Am Acad Child Adolesc Psychiatry 2011;50:583-592.el1.

7) Kim YS, Fombonne E, Koh YJ, Kim SJ, Cheon KA, Leventhal BL. A comparison of DSM-IV pervasive developmental disorder and DSM-5 autism spectrum disorder prevalence in an epidemiologic sample. J Am Acad Child Adolesc Psychiatry 2014;53:500-508.

8) Le Couteur A, Lord C, Rutter M. The autism diagnostic interviewrevised (ADI-R). Los Angeles: Western Psychological Services; 2003.

9) Lord C, Rutter M, Goode S, Heemsbergen J, Jordan H, Mawhood 
L, et al. Autism diagnostic observation schedule: a standardized observation of communicative and social behavior. J Autism Dev Disord 1989;19:185-212.

10) Park H, Gwak G, Park G. K-WPPSI manual. Seoul: Special Education;1995.

11) Kwak K, Park H, Kim C. Korean Wechsler intelligence scale for children-III (K-WISC-III). Seoul: Seoul Special Education Publishing $\mathrm{Co} ; 2001$.

12) Kwak KJ, Oh SW, Kim CT. Korean-Wechsler Intelligence Scale for Children. 4th ed. Seoul: Hakjisa;2011.

13) Yeom T, Park Y, Oh K, Kim J, Lee Y. K-WAIS manual. Seoul: Korea Guidance;1992.

14) Tanguay PE. Autism in DSM-5. Am J Psychiatry 2011;168:1142-1144.

15) McPartland JC, Reichow B, Volkmar FR. Sensitivity and specifici- ty of proposed DSM-5 diagnostic criteria for autism spectrum disorder. J Am Acad Child Adolesc Psychiatry 2012;51:368-383.

16) Wilson CE, Gillan N, Spain D, Robertson D, Roberts G, Murphy CM, et al. Comparison of ICD-10R, DSM-IV-TR and DSM-5 in an adult autism spectrum disorder diagnostic clinic. J Autism Dev Disord 2013:43:2515-2525.

17) Maenner MJ, Rice CE, Arneson CL, Cunniff C, Schieve LA, Carpenter LA, et al. Potential impact of DSM- 5 criteria on autism spectrum disorder prevalence estimates. JAMA Psychiatry 2014;71: 292-300.

18) Swineford LB, Thurm A, Baird G, Wetherby AM, Swedo S. Social (pragmatic) communication disorder: a research review of this new DSM-5 diagnostic category. J Neurodev Disord 2014;6:41. 